\title{
Green-Schwarz superstring on doubled-yet-gauged spacetime
}

\author{
Jeong-Hyuck Park \\ Department of Physics, Sogang University, \\ 35 Baekbeom-ro, Mapo-gu, Seoul 0410\%, Korea \\ B.W. Lee Center for Fields, Gravity and Strings, Institute for Basic Science, \\ Daejeon 34047, Korea \\ E-mail: park@sogang.ac.kr
}

ABSTRACT: We construct a world-sheet action for Green-Schwarz superstring in terms of doubled-yet-gauged spacetime coordinates. For an arbitrarily curved NS-NS background, the action possesses $\mathrm{O}(10,10)$ T-duality, $\operatorname{Spin}(1,9) \times \operatorname{Spin}(9,1)$ Lorentz symmetry, coordinate gauge symmetry, spacetime doubled-yet-gauged diffeomorphisms, world-sheet diffeomorphisms and Weyl symmetry. Further, restricted to flat backgrounds, it enjoys maximal spacetime supersymmetry and kappa-symmetry. After the auxiliary coordinate gauge symmetry potential being integrated out, our action can consistently reduce to the original undoubled Green-Schwarz action. Thanks to the twofold spin groups, the action is unique: it is specific choices of the NS-NS backgrounds that distinguish IIA or IIB, as well as lead to non-Riemannian or non-relativistic superstring a la Gomis-Ooguri which might deserve the nomenclature, type IIC.

Keywords: Superstrings and Heterotic Strings, String Duality, Supersymmetry and Duality

ARXIV EPRINT: 1609.04265 


\section{Contents}

1 Introduction: doubled-yet-gauged 1

2 Green-Schwarz superstring in terms of doubled-yet-gauged coordinates 5

2.1 Main result 5

$\begin{array}{lll}2.2 & \text { Conventions and field contents } & 7\end{array}$

2.3 Target-spacetime supersymmetry and Wess-Zumino term 8

$\begin{array}{lll}2.4 & \text { Fermionic kappa-symmetry } & 9\end{array}$

3 Reductions to type IIA, IIB or IIC $\quad 11$

3.1 Type IIA or IIB: Riemannian backgrounds 12

3.2 Type IIC: non-Riemannian and non-relativistic backgrounds 14

4 Discussion $\quad 16$

$\begin{array}{ll}\text { A Useful identities } & 16\end{array}$

\section{Introduction: doubled-yet-gauged}

Ever since General Relativity was established, it has been customary to adopt Riemannian geometry as the mathematical framework to construct a theory of fundamental physics, such as gravity and string theory. Accordingly, the Riemannian metric, $g_{\mu \nu}$, has been privileged to be the only geometric object which should characterize the nature of gravity. All other fields are viewed as additional 'matters' which live on the geometric background and, at the same time, source the gravitational field.

However, in string theory, the metric is only one segment of the massless NS-NS sector which further includes a two-form gauge potential, $B_{\mu \nu}$, and a scalar dilaton, $\phi$. Under T-duality the three NS-NS fields transform to each other $[1,2]$. This may well imply an alternative gravitational theory where the whole massless NS-NS sector becomes geometric as the gravitational unity. Namely, the three fields, $\left\{g_{\mu \nu}, B_{\mu \nu}, \phi\right\}$, ought to be the trinity of 'stringy gravity'. After series of pioneering works on doubled sigma models [3-8] and Double Field Theory (DFT) [9-11], such an idea has been materialized recently.

First of all, the number of the spacetime coordinates is doubled from $D$ to $D+D$ [3], by adding dual coordinates, $\tilde{x}_{\mu}$, to the conventional ones, $x^{\mu}$, to form doubled $(D+D)$ dimensional coordinates,

$$
x^{M}=\left(\tilde{x}_{\mu}, x^{\nu}\right), \quad M=1,2,3, \cdots, D+D .
$$


On the doubled coordinate space, T-duality becomes a run-of-the-mill $\mathrm{O}(D, D)$ rotation. $^{1}$ However, despite of the doubling, the physical dimension of the spacetime should be undoubled: the doubled coordinates must describe $D$-dimensional physics. One governing geometric principle, proposed in [13] and pursued in this work, is the notion of doubledyet-gauged coordinate system: the doubled coordinate space is gauged by an equivalence relation, called coordinate gauge symmetry,

$$
x^{M} \sim x^{M}+\mathcal{J}^{M N} \Phi_{\mathrm{s}} \partial_{N} \Phi_{\mathrm{t}},
$$

such that it is a gauge orbit that represents a single physical point. Hereafter, $\Phi_{\mathrm{s}}, \Phi_{\mathrm{t}}$ and $\Phi_{\mathrm{u}}$ denote arbitrary fields and their arbitrary derivative descendants which must belong to the theory employing the doubled-yet-gauged coordinate system. Further, $\mathcal{J}^{M N}$ is the inverse of the $\mathrm{O}(D, D)$ invariant metric,

$$
\mathcal{J}_{M N}=\left(\begin{array}{ll}
0 & 1 \\
1 & 0
\end{array}\right), \quad \mathcal{J}^{L M} \mathcal{J}_{M N}=\delta^{L}{ }_{N},
$$

which can freely raise and lower the $\mathrm{O}(D, D)$ vector indices, e.g. $\mathcal{J}^{M N} \partial_{N}=\partial^{M}$.

In Double Field Theory, the equivalence relation, (1.2), is realized by requiring that all the fields in the theory are invariant under the coordinate gauge symmetry shift,

$$
\Phi_{\mathrm{u}}(x)=\Phi_{\mathrm{u}}(x+\Delta), \quad \Delta^{M}=\Phi_{\mathrm{s}} \partial^{M} \Phi_{\mathrm{t}} .
$$

This invariance is then equivalent, i.e. necessary [13] and sufficient [14], to the 'section condition' $[10],{ }^{2}$

$$
\partial_{A} \Phi_{\mathrm{s}} \partial^{A} \Phi_{\mathrm{t}}=0, \quad \partial_{A} \partial^{A} \Phi_{\mathrm{u}}=0,
$$

which are the differential constraints required for the consistency of DFT. ${ }^{3}$ Upon the section condition, the generalized Lie derivatives given by $[10,18]$ (cf. $[19,20]$ ),

$\hat{\mathcal{L}}_{\mathcal{V}} T_{M_{1} \cdots M_{n}}:=\mathcal{V}^{N} \partial_{N} T_{M_{1} \cdots M_{n}}+\omega \partial_{N} \mathcal{V}^{N} T_{M_{1} \cdots M_{n}}+\sum_{i=1}^{n}\left(\partial_{M_{i}} \mathcal{V}_{N}-\partial_{N} \mathcal{V}_{M_{i}}\right) T_{M_{1} \cdots M_{i-1}{ }^{N}{ }_{M_{i+1} \cdots M_{n}}}$

are closed under commutations:

$$
\left[\hat{\mathcal{L}}_{\mathcal{U}}, \hat{\mathcal{L}}_{\mathcal{V}}\right]=\hat{\mathcal{L}}_{[\mathcal{U}, \mathcal{V}]_{\mathrm{C}}}, \quad[\mathcal{U}, \mathcal{V}]_{\mathrm{C}}^{M}:=\mathcal{U}^{N} \partial_{N} \mathcal{V}^{M}-\mathcal{V}^{N} \partial_{N} \mathcal{U}^{M}+\frac{1}{2} \mathcal{V}^{N} \partial^{M} \mathcal{U}_{N}-\frac{1}{2} \mathcal{U}^{N} \partial^{M} \mathcal{V}_{N}
$$

That is to say, the generalized Lie derivative generates the diffeomorphisms on the doubledyet-gauged coordinate system (see [13, 21-26] for finite transformations). Then, in a parallel manner to Riemannian geometry, by taking the whole massless NS-NS sector as the

\footnotetext{
${ }^{1}$ Yet, we stress that the doubled coordinates are not restricted to the description of strings but equally applicable to point-like particle dynamics, see e.g. [12].

${ }^{2}$ The equivalence basically follows from the power series expansion of (1.4). It is worth while to note that the former (strong) constraint in (1.5) implies the latter (weak) one, since $\partial_{A} \partial^{B} \Phi_{\mathrm{s}} \partial_{B} \partial^{C} \Phi_{\mathrm{s}}=0$ means that $\partial_{A} \partial^{B} \Phi_{\mathrm{s}}$ is a nilpotent matrix and hence is traceless. On the other hand, replacing $\Phi_{\mathrm{u}}$ by the product, $\Phi_{\mathrm{s}} \Phi_{\mathrm{t}}$, the latter gives the former.

${ }^{3}$ Yet, cf. [15-17] for the discussion on alternative constraints.
} 
geometric fields, the relevant torsion-free diffeomorphism connection (i.e. "Christoffel symbols"), covariant derivatives, a two-indexed curvature (i.e. "Ricci curvature") and a scalar curvature have been constructed [27] (cf. [28]). ${ }^{4}$ By now, the formalism has been well developed, such that $D=10$ maximally supersymmetric DFT has been constructed to the full order in fermions [30], and the Standard Model itself has been 'double-field-theorized' to covariantly couple to the massless NS-NS sector of the gravitational DFT [31] (cf. [32-36] for related earlier works). In particular, the maximally supersymmetric DFT not only contains and unifies type IIA and IIB supergravities but can also feature 'non-Riemannian' geometry, as we review below.

The massless NS-NS sector enters (bosonic) DFT in the form of a symmetric $\mathrm{O}(D, D)$ element, called "generalized metric",

$$
\mathcal{H}_{M N}=\mathcal{H}_{N M}, \quad \mathcal{H}_{K}{ }^{L} \mathcal{H}_{M}{ }^{N} \mathcal{J}_{L N}=\mathcal{J}_{K M},
$$

along with a scalar density, $e^{-2 d}$, having the weight of unity. Combined with the $\mathrm{O}(D, D)$ invariant metric, the generalized metric can produce a pair of orthogonal and complete symmetric projectors,

$$
\begin{array}{llc}
P_{M N}=P_{N M}=\frac{1}{2}\left(\mathcal{J}_{M N}+\mathcal{H}_{M N}\right), & P_{L}^{M} P_{M}{ }^{N}=P_{L}{ }^{N}, & P_{K}{ }^{L} \bar{P}_{L}{ }^{M}=0, \\
\bar{P}_{M N}=\bar{P}_{N M}=\frac{1}{2}\left(\mathcal{J}_{M N}-\mathcal{H}_{M N}\right), & \bar{P}_{L}{ }^{M} \bar{P}_{M}{ }^{N}=\bar{P}_{L}{ }^{N}, & P_{M N}+\bar{P}_{M N}=\mathcal{J}_{M N} .
\end{array}
$$

These $\mathrm{O}(D, D)$ covariant variables may be generically parametrized in terms of the conventional variables, $\left\{g_{\mu \nu}, B_{\mu \nu}, \phi\right\}$, but there are also exceptions which do not allow such parametrization even locally at all. This leads to the notion of 'non-Riemannian' backgrounds [14, 37] (cf. [38]).

The unification of IIA and IIB is due to the facts that i) the local Lorentz spin group in DFT is twofold, $\operatorname{Spin}(1, D-1) \times \operatorname{Spin}(D-1,1)$ (basically one for $P_{M N}$ and the other for $\left.\bar{P}_{M N}\right)$, ii) the maximally supersymmetric DFT is chiral with respect to both spin groups, $\operatorname{Spin}(1,9)$ and $\operatorname{Spin}(9,1)$, iii) hence, the theory is unique: it admits IIA, IIB and nonRiemannian backgrounds as different types of solutions. In this sense, the last type might deserve the nomenclature, type IIC.

On the other hand, in doubled sigma models where the doubled coordinates are dynamical, the coordinate gauge symmetry (1.2) calls for the relevant gauge connection rather explicitly [14],

$$
D X^{M}:=\mathrm{d} X^{M}-\mathcal{A}^{M} .
$$

As in any gauge theory, the gauge potential, $\mathcal{A}^{M}$, should meet precisely the same property as the gauge generator which is, in the present case, $\Delta^{M}$ in (1.4). Hence, similarly to the section condition (1.5), the coordinate gauge symmetry potential satisfies

$$
\mathcal{A}^{M} \partial_{M}=0, \quad \mathcal{A}^{M} \mathcal{A}_{M}=0 .
$$

Respecting these constraints, the coordinate gauge symmetry is realized as

$$
\delta_{\text {C.G. }} X^{M}=\Phi_{\mathrm{S}} \partial^{M} \Phi_{\mathrm{t}}, \quad \delta_{\text {C.G. }} \mathcal{A}^{M}=\mathrm{d}\left(\Phi_{\mathrm{s}} \partial^{M} \Phi_{\mathrm{t}}\right), \quad \delta_{\text {C.G. }}\left(D X^{M}\right)=0 .
$$

\footnotetext{
${ }^{4}$ Yet, there appears no four-indexed ("Riemann") curvature [29].
} 
Further, while $\mathrm{d} X^{M}$ is not a diffeomorphism covariant vector, $D X^{M}$ is so:

$$
\begin{aligned}
& \delta_{\mathcal{V}} X^{M}=\mathcal{V}^{M}, \quad \delta_{\mathcal{V}}\left(\mathrm{d} X^{M}\right)=\mathrm{d} X^{N} \partial_{N} \mathcal{V}^{M}, \quad \delta_{\mathcal{V}}\left(D X^{M}\right)=\left(\partial_{N} \mathcal{V}^{M}-\partial^{M} \mathcal{V}_{N}\right) D X^{N} \\
& \delta_{\mathcal{V}} \mathcal{A}^{M}=\left(\partial_{N} \mathcal{V}^{M}-\partial^{M} \mathcal{V}_{N}\right) \mathcal{A}^{N}+\partial^{M} \mathcal{V}_{N} \mathrm{~d} X^{N}=-\partial^{M} \mathcal{V}_{N} \mathcal{A}^{N}+\partial^{M} \mathcal{V}_{N} \mathrm{~d} X^{N}
\end{aligned}
$$

It is this gauged one-form, $D X^{M}$, with the obvious kinetic term, $D X^{M} D X^{N} \mathcal{H}_{M N}(X)$, that can be used to construct $\mathrm{O}(D, D)$ T-duality, diffeomorphisms and coordinate gauge symmetry covariant sigma models:

i) world-sheet action for a string [14],

$$
\mathcal{S}_{\text {string }}=\frac{1}{4 \pi \alpha^{\prime}} \int \mathrm{d}^{2} \sigma\left[-\frac{1}{2} \sqrt{-h} h^{i j} D_{i} X^{M} D_{j} X^{N} \mathcal{H}_{M N}(X)-\epsilon^{i j} D_{i} X^{M} \mathcal{A}_{j M}\right],
$$

ii) world-line action for a point-like particle [12],

$$
\mathcal{S}_{\text {particle }}=\int \mathrm{d} \tau\left[e^{-1} D_{\tau} X^{M} D_{\tau} X^{N} \mathcal{H}_{M N}(X)-\frac{1}{4} m^{2} e\right] .
$$

The former result (1.14) was essentially a re-derivation of the doubled string action proposed by Hull [8], with the coordinate gauge symmetry interpretation added. Especially upon Riemannian backgrounds, the Euler-Lagrangian equation of the coordinate gauge symmetry potential, $\mathcal{A}_{i M}$, implies the self-duality (i.e. chirality) over the entire doubled spacetime, cf. (3.20),

$$
D_{i} X_{M}+\frac{1}{\sqrt{-h}} \epsilon_{i}{ }^{j} \mathcal{H}_{M}{ }^{N} D_{j} X_{N}=0,
$$

and the Euler-Lagrangian equation of $X^{M}$ gets simplified to give the stringy geodesic equation,

$$
\frac{1}{\sqrt{-h}} \partial_{i}\left(\sqrt{-h} D^{i} X^{M} \mathcal{H}_{M L}\right)+\Gamma_{L M N}\left(\bar{P} D_{i} X\right)^{M}\left(P D^{i} X\right)^{N}=0,
$$

where $\Gamma_{L M N}$ is the stringy Christoffel connection obtained in [27], and $\left(\bar{P} D_{i} X\right)^{M}=$ $\bar{P}^{M}{ }_{N} D_{i} X^{N}$ etc. It is worth while to note that the world-sheet topological term in (1.14) transforms to total derivatives under the coordinate gauge symmetry (1.12) as well as under the diffeomorphisms (1.13) [14],

$$
\delta_{\text {C.G. }}\left(\epsilon^{i j} D_{i} X^{M} \mathcal{A}_{j M}\right)=-\partial_{i}\left(\epsilon^{i j} \Phi_{\mathrm{s}} \partial_{j} \Phi_{\mathrm{t}}\right), \quad \delta_{\mathcal{V}}\left(\epsilon^{i j} D_{i} X^{M} \mathcal{A}_{j M}\right)=\partial_{i}\left(\epsilon^{i j} \mathcal{V}_{M} \partial_{j} X^{M}\right) .
$$

The kinetic terms in (1.14) and (1.15) are invariant under the coordinate gauge symmetry, and they transform 'covariantly' under the diffeomorphisms, $\delta_{\mathcal{V}}\left(D X^{M} D X^{N} \mathcal{H}_{M N}\right)=$ $D X^{M} D X^{N} \hat{\mathcal{L}}_{\mathcal{V}} \mathcal{H}_{M N}$, such that any Killing vector satisfying $\hat{\mathcal{L}}_{\mathcal{V}} \mathcal{H}_{M N}=0$ induces a Noether symmetry of the action.

In the above doubled sigma models, the gauge potentials are all auxiliary. After they are integrated out, the doubled sigma models consistently reduce to the conventional undoubled string and particle actions.

It is the purpose of the present paper to supersymmetrize the above doubled string action (1.14), or equivalently to formulate the renowned Green-Schwarz superstring action [39] on the doubled-yet-gauged spacetime, as the complementary world-sheet counterpart to the maximally supersymmetric DFT [30]. 
Our constructed action is going to be symmetric, with respect to

- $\mathrm{O}(10,10)$ T-duality,

- $\operatorname{Spin}(1,9) \times \operatorname{Spin}(9,1)$ Lorentz symmetry,

- coordinate gauge symmetry,

- target-spacetime doubled-yet-gauged diffeomorphisms (over Killing directions),

- world-sheet diffeomorphisms,

- conformal symmetry under Weyl transformations,

and, in addition, restricted to flat NS-NS backgrounds,

- target-spacetime 16+16 global supersymmetry,

- 16+16 kappa-symmetry.

Since we do not include spin connections, the $\operatorname{Spin}(1,9) \times \operatorname{Spin}(9,1)$ Lorentz symmetry is going to be global rather than local. Nevertheless, the global twofold spin structure ensures to unify IIA and IIB superstrings: different choices of the NS-NS backgrounds give rise to IIA or IIB, as well as non-Riemannian IIC superstrings. Once again, after the auxiliary coordinate gauge symmetry potential being integrated out, our action reduces consistently to the Green-Schwarz type IIA/B superstring action if the background is Riemannian. Alternatively, upon a non-Riemannian background, our action leads to the supersymmetric extension of the Gomis-Ooguri non-relativistic string [38].

For further inspiring precursors, we refer readers to $[8,40]$ for the world-sheet supersymmetries, [41] for the construction of chiral affine (super-)Lie algebras, [42] for the T-duality supergroup, $\operatorname{OSp}(D, D \mid 2 s)$, as well as [43] for a doubled Hamiltonian sigma model and $[44,45]$ for the Born reciprocity. We also refer the work by Bandos [46, 47] on the construction of a PST superstring action in doubled superspace.

\section{Green-Schwarz superstring in terms of doubled-yet-gauged coordi- nates}

In this section, firstly we present our main result, i.e. 'the construction of the GreenSchwarz superstring action on the doubled-yet-gauged spacetime', and then provide the relevant explanations, such as the conventions, the field contents, the target-spacetime supersymmetry and the kappa-symmetry. The reductions to the undoubled type IIA, IIB and non-relativistic IIC superstrings will be discussed in the next section.

\subsection{Main result}

We propose the Green-Schwarz superstring action on the doubled-yet-gauged spacetime,

$$
\mathcal{S}_{\text {superstring }}=\frac{1}{4 \pi \alpha^{\prime}} \int \mathrm{d}^{2} \sigma \mathcal{L}_{\text {superstring }},
$$


with the Lagrangian,

$$
\mathcal{L}_{\text {superstring }}=-\frac{1}{2} \sqrt{-h} h^{i j} \Pi_{i}^{M} \Pi_{j}^{N} \mathcal{H}_{M N}-\epsilon^{i j} D_{i} X^{M}\left(\mathcal{A}_{j M}-i \Sigma_{j M}\right) .
$$

Here, equipped with the map from the string world-sheet to the doubled-yet-gauged targetspacetime,

$$
\sigma^{i} \longrightarrow X^{M}=\left(\widetilde{X}_{\mu}, X^{\nu}\right)
$$

and a pair of Majorana-Weyl spinors, $\theta^{\alpha}$ for $\operatorname{Spin}(1,9)$ and $\theta^{\prime \bar{\alpha}}$ for $\operatorname{Spin}(9,1)$, we set

$$
\Pi_{i}^{M}:=D_{i} X^{M}-i \Sigma_{i}^{M}, \quad \Sigma_{i}^{M}:=\bar{\theta} \gamma^{M} \partial_{i} \theta+\bar{\theta}^{\prime} \bar{\gamma}^{M} \partial_{i} \theta^{\prime} .
$$

For an arbitrarily curved NS-NS background, the action possesses the manifest $\mathrm{O}(10,10)$ T-duality, the $\operatorname{Spin}(1,9) \times \operatorname{Spin}(9,1)$ global Lorentz symmetry, the coordinate gauge symmetry, the target-spacetime doubled-yet-gauged diffeomorphisms over any Killing direction, world-sheet diffeomorphisms and Weyl symmetry.

Moreover, when the background is flat, the action is invariant under $16+16$ global target-spacetime supersymmetry,

$$
\delta_{\varepsilon} X^{M}=i \bar{\varepsilon} \gamma^{M} \theta+i \bar{\varepsilon}^{\prime} \bar{\gamma}^{M} \theta^{\prime}, \quad \delta_{\varepsilon} \theta=\varepsilon, \quad \delta_{\varepsilon} \theta^{\prime}=\varepsilon^{\prime}, \quad \delta_{\varepsilon} h_{i j}=0, \quad \delta_{\varepsilon} \mathcal{A}_{i M}=0,
$$

as well as 16+16 local fermionic kappa-symmetry,

$$
\begin{aligned}
\delta_{\kappa} X^{M} & =i \bar{\theta} \gamma^{M} \delta_{\kappa} \theta+i \bar{\theta}^{\prime} \bar{\gamma}^{M} \delta_{\kappa} \theta^{\prime}, \quad \delta_{\kappa} \theta=h_{+}^{i j} \Pi_{i M} \gamma^{M} \kappa_{j}, \quad \delta_{\kappa} \theta^{\prime}=h_{-}^{i j} \Pi_{i M} \bar{\gamma}^{M} \kappa_{j}^{\prime}, \\
\delta_{\kappa}\left(\sqrt{-h} h^{i j}\right) & =-8 i \sqrt{-h}\left(h_{+}^{i k} h_{+}^{j l} \partial_{k} \bar{\theta} \kappa_{l}+h_{-}^{i k} h_{-}^{j l} \partial_{k} \bar{\theta}^{\prime} \kappa_{l}^{\prime}\right) \\
\delta_{\kappa} \mathcal{A}_{i M} & =-2 i\left(h_{+i}{ }^{j} h_{+}^{k l} \partial_{j} \bar{\theta} \kappa_{l}+h_{-i}{ }^{j} h_{-}^{k l} \partial_{j} \bar{\theta}^{\prime} \kappa_{l}^{\prime}\right)\left[\widehat{\Pi}_{k M}\right]_{\text {projected }}
\end{aligned}
$$

In the above, we set a pair of world-sheet projection matrices,

$$
h_{+}^{i j}:=\frac{1}{2}\left(h^{i j}+\frac{\epsilon^{i j}}{\sqrt{-h}}\right), \quad h_{-}^{i j}:=\frac{1}{2}\left(h^{i j}-\frac{\epsilon^{i j}}{\sqrt{-h}}\right)=h_{+}^{j i},
$$

and the self-dual part of $\Pi_{i}^{M}$,

$$
\widehat{\Pi}_{i}^{M}:=\Pi_{i}^{M}+\frac{\epsilon_{i}{ }^{j}}{\sqrt{-h}} \mathcal{H}^{M}{ }_{N} \Pi_{j}^{N} .
$$

Further, $\left[\widehat{\Pi}_{k M}\right]_{\text {projected }}$ means the projection of $\widehat{\Pi}_{k M}$ to the coordinate gauge symmetry value, such that

$$
\left[\widehat{\Pi}_{k}^{M}\right]_{\text {projected }} \times \partial_{M}=0 .
$$

Concretely, without loss of generality up to $\mathrm{O}(10,10)$ rotations, if we choose the section as

$$
\partial_{M}=\left(\tilde{\partial}^{\mu}, \partial_{\nu}\right) \equiv\left(0, \partial_{\nu}\right), \quad \mathcal{A}_{i M} \equiv\left(0, A_{i \mu}\right),
$$

we have

$$
\left[\widehat{\Pi}_{i}^{M}\right]_{\text {projected }}=\left[\left(\widetilde{\widehat{\Pi}}_{i \mu}, \widehat{\Pi}_{i}^{\nu}\right)\right]_{\text {projected }} \equiv\left(\widetilde{\widehat{\Pi}}_{i \mu}, 0\right)
$$




\begin{tabular}{|c|c|c|}
\hline Index & Representation & Raising \& Lowering Indices \\
\hline$M, N, \cdots$ & Target-space diffeomorphism \& $\mathrm{O}(10,10)$ vector & $\mathcal{J}_{A B}(1.3)$ \\
$p, q, \cdots$ & $\operatorname{Spin}(1,9)$ vector & $\eta_{p q}=\operatorname{diag}(-++\cdots+)$ \\
$\alpha, \beta, \cdots$ & $\operatorname{Spin}(1,9)$ spinor & $C_{\alpha \beta}=C_{\beta \alpha},\left(\gamma^{p}\right)^{T}=C \gamma^{p} C^{-1}(2.13)$ \\
$\bar{p}, \bar{q}, \cdots$ & $\operatorname{Spin}(9,1)$ vector & $\bar{\eta}_{\bar{p} \bar{q}}=\operatorname{diag}(+--\cdots-)$ \\
$\bar{\alpha}, \bar{\beta}, \cdots$ & $\operatorname{Spin}(9,1)$ spinor & $\bar{C}_{\bar{\alpha} \bar{\beta}}=\bar{C}_{\bar{\beta} \bar{\alpha}},\left(\bar{\gamma}_{\bar{p}}\right)^{T}=\bar{C} \bar{\gamma}^{\bar{p}} \bar{C}^{-1}(2.13)$ \\
$i, j, \cdots$ & World-sheet diffeomorphism vector & $h_{i j}$ \\
\hline
\end{tabular}

Table 1. Convention of the indices and the corresponding "metric" to raise or lower the positions.

and thus,

$$
\delta_{\kappa} \mathcal{A}_{i M}=\left(0, \delta_{\kappa} A_{i \mu}\right)=-2 i\left(h_{+i}{ }^{j} h_{+}^{k l} \partial_{j} \bar{\theta} \kappa_{l}+h_{-i}{ }^{j} h_{-}^{k l} \partial_{j} \bar{\theta}^{\prime} \kappa_{l}^{\prime}\right) \times\left(0, \widetilde{\Pi}_{k \mu}\right)
$$

Surely, $\varepsilon, \varepsilon^{\prime}$ are constant Majorana-Weyl $\operatorname{Spin}(1,9)$, $\operatorname{Spin}(9,1)$ spinors having the same chiralities as $\theta, \theta^{\prime}$ respectively, while $\kappa_{i}, \kappa_{j}^{\prime}$ are local Majorana-Weyl spinors with the opposite chiralities. As stressed by Hull [8], the string tension on a doubled space should be halved, i.e. $\left(4 \pi \alpha^{\prime}\right)^{-1}$. Further explanations are in order in the following subsections.

\subsection{Conventions and field contents}

Our conventions, especially for the indices, are identical to $[30,36]$ and summarized in table 1.

Since the Spin group is twofold as $\operatorname{Spin}(1,9) \times \operatorname{Spin}(9,1)$, there exist a pair of gamma matrices, $\left(\gamma^{p}\right)^{\alpha}{ }_{\beta}$ and $\left(\bar{\gamma}^{\bar{p}}\right)^{\bar{\alpha}} \bar{\beta}$. The corresponding charge conjugation matrices, $C_{\alpha \beta}$ and $\bar{C}_{\bar{\alpha} \bar{\beta}}$, satisfy for $n=0,1,2, \cdots$,

$$
\begin{aligned}
&\left(C \gamma^{p_{1} p_{2} \cdots p_{n}}\right)_{\alpha \beta}=(-1)^{n(n-1) / 2}\left(C \gamma^{p_{1} p_{2} \cdots p_{n}}\right)_{\beta \alpha}, \\
&\left(\bar{C} \bar{\gamma}^{\bar{p}_{1} \bar{p}_{2} \cdots \bar{p}_{n}}\right)_{\bar{\alpha} \bar{\beta}}=(-1)^{n(n-1) / 2}\left(\bar{C} \bar{\gamma}^{\bar{p}_{1} \bar{p}_{2} \cdots \bar{p}_{n}}\right)_{\bar{\beta} \bar{\alpha}} .
\end{aligned}
$$

A well-known crucial Fierz identity is

$$
\left(C \gamma^{p} \gamma_{+}\right)_{(\alpha \beta}\left(C \gamma_{p} \gamma_{+}\right)_{\gamma) \delta}=0, \quad\left(\bar{C} \bar{\gamma}^{\bar{p}} \bar{\gamma}_{+}\right)_{(\bar{\alpha} \bar{\beta}}\left(\bar{C} \bar{\gamma}_{\bar{p}} \bar{\gamma}_{+}\right)_{\bar{\gamma}) \bar{\delta}}=0
$$

where $\gamma_{+}, \bar{\gamma}_{+}$denote the usual chiral projection matrices,

$$
\begin{aligned}
& \gamma_{+}:=\frac{1}{2}\left[1+\gamma^{(11)}\right], \quad \gamma^{(11)}:=\gamma^{012 \cdots 9}, \quad\left(\gamma_{+}\right)^{2}=\gamma_{+}, \quad\left(\gamma^{(11)}\right)^{2}=1, \\
& \bar{\gamma}_{+}:=\frac{1}{2}\left[1+\bar{\gamma}^{(11)}\right], \quad \bar{\gamma}^{(11)}:=\bar{\gamma}^{012 \cdots 9}, \quad\left(\bar{\gamma}_{+}\right)^{2}=\bar{\gamma}_{+}, \quad\left(\bar{\gamma}^{(11)}\right)^{2}=1 .
\end{aligned}
$$

The NS-NS background of the action is given by the DFT-vielbeins satisfying four defining properties:

$$
V_{M p} V_{q}^{M}=\eta_{p q}, \quad \bar{V}_{M \bar{p}} \bar{V}_{\bar{q}}^{M}=\bar{\eta}_{\bar{p} \bar{q}}, \quad V_{M p} \bar{V}_{\bar{q}}^{M}=0, \quad V_{M p} V_{N}^{p}+\bar{V}_{M \bar{p}} \bar{V}_{N} \bar{p}=\mathcal{J}_{M N} .
$$


That is to say, they are normalized, orthogonal and complete. They correspond to the "square-roots" of the projectors (1.9), as

$$
P_{M N}=V_{M p} V_{N}^{p}, \quad \bar{P}_{M N}=\bar{V}_{M \bar{p}} \bar{V}_{N}^{\bar{p}},
$$

while the generalized metric is given by the difference,

$$
\mathcal{H}_{M N}=V_{M p} V_{N}^{p}-\bar{V}_{M \bar{p}} \bar{V}_{N}^{\bar{p}} .
$$

It follows then that

$$
\mathcal{H}_{M}{ }^{N} V_{N p}=+V_{M p}, \quad \mathcal{H}_{M}{ }^{N} \bar{V}_{N \bar{p}}=-\bar{V}_{M \bar{p}}
$$

In this way, the DFT-vielbeins simultaneously diagonalize $\mathcal{J}_{M N}$ and $\mathcal{H}_{M N}$ into ' $\operatorname{diag}(\eta, \bar{\eta})$ ' and ' $\operatorname{diag}(\eta,-\bar{\eta})$ ' respectively. As a solution to (2.16), they may be parametrized generically by ordinary zehnbeins and $B$-field (3.2), (up to field redefinitions, e.g. [50]); or they may not admit such a conventional i.e. Riemannian parametrization [14, 37].

Contracted with the DFT-vilebeins, the gamma matrices can carry $\mathrm{O}(10,10)$ vector indices, such as $\gamma^{M}=V_{p}^{M} \gamma^{p}$ and $\bar{\gamma}^{M}=\bar{V}^{M}{ }_{\bar{p}} \bar{\gamma}^{\bar{p}}$ which satisfy then

$$
\gamma^{M} \gamma^{N}+\gamma^{N} \gamma^{M}=2 P^{A B}, \quad \bar{\gamma}^{M} \bar{\gamma}^{N}+\bar{\gamma}^{N} \bar{\gamma}^{M}=2 \bar{P}^{A B}
$$

All the spinors are fermionic Majorana-Weyl spinors for either the $\operatorname{Spin}(1,9)$ or the $\operatorname{Spin}(9,1)$ Lorentz group, in particular to meet

$$
\theta=\gamma^{(11)} \theta, \quad \bar{\theta}=\theta^{T} C=-\bar{\theta} \gamma^{(11)}, \quad \theta^{\prime}=\bar{\gamma}^{(11)} \theta^{\prime}, \quad \bar{\theta}^{\prime}=\theta^{T} \bar{C}=-\bar{\theta}^{\prime} \bar{\gamma}^{(11)} .
$$

It is worth while to note that, using the properties of the coordinate gauge symmetry potential (1.11), we may rewrite the world-sheet topological term as

$$
\epsilon^{i j} D_{i} X^{M}\left(\mathcal{A}_{j M}-i \Sigma_{j M}\right)=\epsilon^{i j}\left(\Pi_{i}^{M} \mathcal{A}_{j M}-i \partial_{i} X^{M} \Sigma_{j M}\right) .
$$

\subsection{Target-spacetime supersymmetry and Wess-Zumino term}

For flat NS-NS backgrounds where the DFT-vielbeins are all constant, $\Pi_{i}^{M}$ is targetspacetime supersymmetry invariant, under (2.5),

$$
\delta_{\varepsilon} \Pi_{i}^{M}=0,
$$

and the Lagrangian transforms to total derivatives, implying the invariance of the action,

$$
\begin{aligned}
\delta_{\varepsilon} \mathcal{L}_{\text {superstring }} & =\epsilon^{i j}\left(\partial_{i} X^{M} \partial_{j} \delta_{\varepsilon} X_{M}+\Sigma_{i}^{M} \delta_{\varepsilon} \Sigma_{j M}\right) \\
& =-\epsilon^{i j} \partial_{i}\left(\partial_{j} X^{M} \delta_{\varepsilon} X_{M}+\frac{1}{3} \bar{\varepsilon} \gamma^{p} \theta \bar{\theta} \gamma_{p} \partial_{j} \theta+\frac{1}{3} \bar{\varepsilon}^{\prime} \bar{\gamma}^{\bar{p}} \theta^{\prime} \bar{\theta}^{\prime} \bar{\gamma}_{\bar{p}} \partial_{j} \theta^{\prime}\right) .
\end{aligned}
$$

In the above, the second equality follows essentially from the Fierz identity (2.14) which enables us to write

$$
\epsilon^{i j} \gamma^{p} \partial_{i} \theta \bar{\theta} \gamma_{p} \partial_{j} \theta=\frac{1}{3} \partial_{i}\left(\epsilon^{i j} \gamma^{p} \theta \bar{\theta} \gamma_{p} \partial_{j} \theta\right), \quad \epsilon^{i j} \bar{\gamma}^{\bar{p}} \partial_{i} \theta^{\prime} \bar{\theta}^{\prime} \bar{\gamma}_{\bar{p}} \partial_{j} \theta^{\prime}=\frac{1}{3} \partial_{i}\left(\epsilon^{i j} \bar{\gamma}^{\bar{p}} \theta^{\prime} \bar{\theta}^{\prime} \bar{\gamma}_{\bar{p}} \partial_{j} \theta^{\prime}\right) .
$$


In fact, extending the two-dimensional world-sheet to a fictitious three-dimensional space and using identities due to (2.14) like

$$
\epsilon^{i j k} \bar{\theta} \gamma^{p} \partial_{i} \theta \partial_{j} \bar{\theta} \gamma_{p} \partial_{k} \theta=0, \quad \epsilon^{i j k} \bar{\theta}^{\prime} \bar{\gamma}^{\bar{p}} \partial_{i} \theta^{\prime} \partial_{j} \bar{\theta}^{\prime} \bar{\gamma}_{\bar{p}} \partial_{k} \theta^{\prime}=0
$$

we may straightforwardly compute the 'exterior derivative' of the topological term,

$$
\epsilon^{i j k} \partial_{k}\left[D_{i} X^{M}\left(\mathcal{A}_{j M}-i \Sigma_{j M}\right)\right]=\epsilon^{i j k} \Pi_{i}^{M}\left(i \partial_{j} \bar{\theta} \gamma_{M} \partial_{k} \theta+i \partial_{j} \bar{\theta}^{\prime} \bar{\gamma}_{M} \partial_{k} \theta^{\prime}-\frac{1}{2} \mathcal{F}_{j k M}\right)
$$

where we set the field strength of the coordinate gauge symmetry potential, $\mathcal{F}_{j k M}:=$ $\partial_{j} \mathcal{A}_{k M}-\partial_{k} \mathcal{A}_{j M}$. The resulting 'three-form' on the right hand side of the equality in (2.27) then corresponds to the Wess-Zumino term [48] for Green-Schwarz superstring [49] now on doubled-yet-gauged spacetime. As desired, it is manifestly invariant under the global target-spacetime supersymmetry (2.5).

\subsection{Fermionic kappa-symmetry}

For the systematic derivation of the kappa-symmetry, we start with generic variations of the spinors, $\delta \theta, \delta \theta^{\prime}$, and the auxiliary fields, $\delta \mathcal{A}_{i M}, \delta h_{i j}$, while we set, with the opposite sign compared to the target-spacetime supersymmetry $(2.5)$,

$$
\delta X^{M}=i \bar{\theta} \gamma^{M} \delta \theta+i \bar{\theta}^{\prime} \bar{\gamma}^{M} \delta \theta^{\prime}=-i \delta \bar{\theta} \gamma^{M} \theta-i \delta \bar{\theta}^{\prime} \bar{\gamma}^{M} \theta^{\prime} .
$$

It follows straightforwardly upon flat backgrounds,

$$
\delta \Pi_{i}^{M}=-2 i \delta \bar{\theta} \gamma^{M} \partial_{i} \theta-2 i \delta \bar{\theta}^{\prime} \bar{\gamma}^{M} \partial_{i} \theta^{\prime}-\delta \mathcal{A}_{i}^{M},
$$

and the kinetic term transforms as

$$
\begin{aligned}
& \delta\left(-\frac{1}{2} \sqrt{-h} h^{i j} \Pi_{i}^{M} \Pi_{j}^{N} \mathcal{H}_{M N}\right) \\
& =-\frac{1}{2} \delta\left(\sqrt{-h} h^{i j}\right) \Pi_{i}^{M} \Pi_{j}^{N} \mathcal{H}_{M N}+\sqrt{-h} h^{i j} \Pi_{i M}\left(2 i \delta \bar{\theta} \gamma^{M} \partial_{j} \theta-2 i \delta \bar{\theta}^{\prime} \bar{\gamma}^{M} \partial_{j} \theta^{\prime}+\mathcal{H}^{M N} \delta \mathcal{A}_{j N}\right) .
\end{aligned}
$$

On the other hand, the Fierz identity (2.25) implies for arbitrary $\delta \theta$ and $\delta \theta^{\prime}$,

$$
\begin{aligned}
& \epsilon^{i j} \partial_{i}\left(\bar{\theta} \gamma^{p} \delta \theta\right) \bar{\theta} \gamma_{p} \partial_{j} \theta=\partial_{i}\left(\epsilon^{i j} \bar{\theta} \gamma^{p} \delta \theta \bar{\theta} \gamma_{p} \partial_{j} \theta\right)+2 \epsilon^{i j} \delta \bar{\theta} \gamma^{p} \partial_{i} \theta \bar{\theta} \gamma_{p} \partial_{j} \theta \\
& \epsilon^{i j} \partial_{i}\left(\bar{\theta}^{\prime} \bar{\gamma}^{\bar{p}} \delta \theta^{\prime}\right) \bar{\theta}^{\prime} \bar{\gamma}_{\bar{p}} \partial_{j} \theta^{\prime}=\partial_{i}\left(\epsilon^{i j} \bar{\theta}^{\prime} \bar{\gamma}^{\bar{p}} \delta \theta^{\prime} \bar{\theta}^{\prime} \bar{\gamma}_{\bar{p}} \partial_{j} \theta^{\prime}\right)+2 \epsilon^{i j} \delta \bar{\theta}^{\prime} \bar{\gamma}^{\bar{p}} \partial_{i} \theta^{\prime} \bar{\theta}^{\prime} \bar{\gamma}_{\bar{p}} \partial_{j} \theta^{\prime}
\end{aligned}
$$

which in turn enable us to organize the variation of the world-sheet topological term as

$$
\begin{aligned}
& \delta\left[-\epsilon^{i j} D_{i} X^{M}\left(\mathcal{A}_{j M}-i \Sigma_{j M}\right)\right] \\
& =2 i \epsilon^{i j} \Pi_{i}{ }^{M}\left(\delta \bar{\theta} \gamma_{M} \partial_{j} \theta+\delta \bar{\theta}^{\prime} \bar{\gamma}_{M} \partial_{j} \theta^{\prime}\right)+\epsilon^{i j} \delta \mathcal{A}_{i M} \Pi_{j}^{M}-\partial_{i}\left[\epsilon^{i j}\left(\Pi_{j}{ }^{M}+\mathcal{A}_{j}^{M}\right) \delta X_{M}\right] .
\end{aligned}
$$

Combining (2.30) and (2.32), with (2.7), (2.8), we obtain

$$
\begin{aligned}
\delta \mathcal{L}_{\text {superstring }}= & -\frac{1}{2} \delta\left(\sqrt{-h} h^{i j}\right) \Pi_{i}^{M} \Pi_{j}^{N} \mathcal{H}_{M N}+4 i \sqrt{-h} \Pi_{i M}\left(h_{+}^{i j} \delta \bar{\theta} \gamma^{M} \partial_{j} \theta-h_{-}^{i j} \delta \bar{\theta}^{\prime} \bar{\gamma}^{M} \partial_{j} \theta^{\prime}\right) \\
& +\epsilon^{i j} \delta \mathcal{A}_{i M} \widehat{\Pi}_{j}^{M}-\partial_{i}\left[\epsilon^{i j}\left(\Pi_{j}{ }^{M}+\mathcal{A}_{j}^{M}\right) \delta X_{M}\right]
\end{aligned}
$$


where the world-sheet projection matrices, $h_{ \pm}^{i j}(2.7)$, naturally appear. They satisfy

$$
h_{+j}^{i} h_{+k}^{j}=h_{+k}^{i}, \quad h_{-j}^{i} h_{-k}^{j}=h_{-k}^{i}, \quad h_{+j}^{i} h_{-k}^{j}=0, \quad h_{+j}^{i}+h_{-j}^{i}=\delta^{i}{ }_{j} .
$$

There are four terms on the right hand side of the equality in (2.33). The last term is total derivative and hence harmless. The first term is quadratic in $\Pi_{i M}$ and needs to be canceled by other two terms (i.e. second and third). For this, the variations of the fermions need to be linear in $\Pi_{i}^{M}$, such as

$$
\delta_{\kappa} \theta=\Pi_{i M} \gamma^{M} \zeta^{i}, \quad \delta_{\kappa} \theta^{\prime}=\Pi_{i M} \bar{\gamma}^{A} \zeta^{\prime i} .
$$

Substituting this ansatz into (2.33), from (A.3), (A.4), (A.6) and through an intermediate step (A.7), the variation of the Lagrangian further reduces to

$$
\begin{aligned}
\delta \mathcal{L}_{\text {superstring }}= & -2 i\left(h_{-j}^{i} \partial_{i} \bar{\theta} \gamma_{M N} \zeta^{j}+h_{+j}^{i} \partial_{i} \bar{\theta}^{\prime} \bar{\gamma}_{M N} \zeta^{\prime j}\right)\left(\epsilon^{k l} \Pi_{k}^{M} \Pi_{l}^{N}\right) \\
& -\frac{1}{2}\left[\delta\left(\sqrt{-h} h^{i j}\right)+8 i \sqrt{-h}\left(\partial_{k} \bar{\theta} \zeta^{(i} h_{+}^{j) k}+\partial_{k} \bar{\theta}^{\prime} \zeta^{\prime(i} h_{-}^{j) k}\right)\right] \Pi_{i}^{M} \Pi_{j}^{N} \mathcal{H}_{M N} \\
& +\epsilon^{i j}\left[\delta \mathcal{A}_{i M}+2 i\left(h_{+i}{ }^{k} \partial_{k} \bar{\theta} \zeta^{l}+h_{-i}{ }^{k} \partial_{k} \bar{\theta}^{\prime} \zeta^{\prime l}\right) \Pi_{l M}\right] \widehat{\Pi}_{j}^{M} \\
& -\partial_{i}\left[\epsilon^{i j}\left(\Pi_{j}{ }^{M}+\mathcal{A}_{j}^{M}\right) \delta X_{M}\right] .
\end{aligned}
$$

Except the last harmless term, each line on the right hand side should vanish by itself. The vanishing of the first line requires

$$
h_{-j}^{i} \zeta^{j}=0, \quad h_{+j}^{i} \zeta^{\prime j}=0 \quad \Longleftrightarrow \quad \zeta^{i}=h_{+}^{i j} \kappa_{j}, \quad \zeta^{\prime i}=h_{-}^{i j} \kappa_{j}^{\prime},
$$

which fix the kappa-symmetry transformations of the fermions, $\delta_{\kappa} \theta, \delta_{\kappa} \theta^{\prime}$, completely as (2.6). Consequently the second line determines the variation of the world-sheet metric (2.6), up to Weyl transformations, which we rewrite here,

$$
\delta_{\kappa}\left(\sqrt{-h} h^{i j}\right)=-8 i \sqrt{-h}\left(h_{+}^{i k} h_{+}^{j l} \partial_{k} \bar{\theta} \kappa_{l}+h_{-}^{i k} h_{-}^{j l} \partial_{k} \bar{\theta}^{\prime} \kappa_{l}^{\prime}\right) .
$$

For consistency with

$$
\delta\left(\sqrt{-h} h^{i j}\right)=-\sqrt{-h}\left(h^{i k} h^{j l}-\frac{1}{2} h^{k l} h^{i j}\right) \delta h_{k l},
$$

the variation $(2.38)$ is, from $(2.34),($ A.4), symmetric and traceless,

$$
\delta_{\kappa}\left(\sqrt{-h} h^{i j}\right)=\delta_{\kappa}\left(\sqrt{-h} h^{j i}\right), \quad h_{i j} \delta_{\kappa}\left(\sqrt{-h} h^{j i}\right)=0 .
$$

With (2.37) and (2.38) assumed, the variation of the Lagrangian spelled in (2.36) simplifies, through some intermediate step (A.8), to

$$
\begin{aligned}
& \delta \mathcal{L}_{\text {superstring }}+\partial_{i}\left[\epsilon^{i j}\left(\Pi_{j}{ }^{M}+\mathcal{A}_{j}^{M}\right) \delta X_{M}\right] \\
& =\epsilon^{i j}\left[\delta \mathcal{A}_{i M}+i\left(h_{+i}{ }^{k} h_{+}^{l m} \partial_{k} \bar{\theta} \kappa_{m}+h_{-i}{ }^{k} h_{-}^{l m} \partial_{k} \bar{\theta}^{\prime} \kappa_{m}^{\prime}\right) \widehat{\Pi}_{l M}\right] \widehat{\Pi}_{j}^{M} .
\end{aligned}
$$

The vanishing of the right hand side of the above equality then should fix the kappasymmetry transformation of the coordinate gauge symmetry potential. Yet, since the 
potential is constrained to satisfy $\mathcal{A}_{i}^{M} \partial_{M}=0$ and $\mathcal{A}_{i}^{M} \mathcal{A}_{j M}=0$ (1.11), it does not take the naive form one might be tempted to put:

$$
\delta \mathcal{A}_{i M} \neq-i\left(h_{+i}{ }^{k} h_{+}^{l m} \partial_{k} \bar{\theta} \kappa_{m}+h_{-i}{ }^{k} h_{-}^{l m} \partial_{k} \bar{\theta}^{\prime} \kappa_{m}^{\prime}\right) \widehat{\Pi}_{l M} .
$$

Instead, we must "double" this and project $\widehat{\Pi}_{l M}$ to the coordinate gauge symmetry value,

$$
\delta_{\kappa} \mathcal{A}_{i M}=-2 i\left(h_{+i}{ }^{k} h_{+}^{l m} \partial_{k} \bar{\theta} \kappa_{m}+h_{-i}{ }^{k} h_{-}^{l m} \partial_{k} \bar{\theta}^{\prime} \kappa_{m}^{\prime}\right)\left[\widehat{\Pi}_{l M}\right]_{\text {projected }},
$$

such that

$$
\left[\widehat{\Pi}_{l}^{M}\right]_{\text {projected }} \times \partial_{M}=0 .
$$

Concretely, the off-block diagonal form of the $\mathrm{O}(10,10)$ invariant metric, $\mathcal{J}_{M N}(1.3)$ naturally decomposes all the doubled variables into two parts, such as

$$
X^{M}=\left(\widetilde{X}_{\mu}, X^{\nu}\right), \quad \partial_{M}=\left(\widetilde{\partial}^{\mu}, \partial_{\nu}\right), \quad \Pi_{i}^{M}=\left(\widetilde{\Pi}_{i \mu}, \Pi_{i}^{\nu}\right), \quad \widehat{\Pi}_{i}^{M}=\left(\widetilde{\Pi}_{i \mu}, \widehat{\Pi}_{i}^{\nu}\right) .
$$

Without loss of generality up to $\mathrm{O}(10,10)$ rotations, if we choose the section by

$$
\partial_{M}=\left(0, \partial_{\nu}\right), \quad \mathcal{A}_{i M} \equiv\left(0, A_{i \mu}\right),
$$

we get

$$
\left[\widehat{\Pi}_{l M}\right]_{\text {projected }} \equiv\left(0, \widetilde{\Pi}_{i \mu}\right),
$$

and thus, the kappa-symmetry transformation of the coordinate gauge symmetry potential (2.43) reads explicitly,

$$
\delta_{\kappa} A_{i \mu}=-2 i\left(h_{+i}{ }^{k} h_{+}^{l m} \partial_{k} \bar{\theta} \kappa_{m}+h_{-i}{ }^{k} h_{-}^{l m} \partial_{k} \bar{\theta}^{\prime} \kappa_{m}^{\prime}\right) \widetilde{\widehat{\Pi}}_{l \mu} .
$$

After all, under the kappa-symmetry, the Lagrangian transforms to the total derivative,

$$
\delta_{\kappa} \mathcal{L}_{\text {superstring }}=-\partial_{i}\left[\epsilon^{i j}\left(\Pi_{j}^{M}+\mathcal{A}_{j}^{M}\right) \delta_{\kappa} X_{M}\right] .
$$

\section{Reductions to type IIA, IIB or IIC}

One of the characteristics in our construction of the superstring action (2.1) - as a counterpart to the maximally supersymmetric DFT [30] — is the usage of not the conventional Riemannian variables, $\left\{g_{\mu \nu}, B_{\mu \nu}, \phi\right\}$, but the $\mathrm{O}(10,10)$ covariant genuine DFT variables: in particular, the DFT-vielbeins. They represent the doubled-yet-gauged spacetime NS-NS background on which the Green-Schwarz superstring propagates. As long as their defining algebraic relations (2.16) are satisfied, our superstring action, as well as the targetspacetime supersymmetric DFT, all work autonomically without resorting to the Riemannian geometry or parametrization. The connection to the conventional Riemannian formulations, such as supergravities and the original Green-Schwarz superstring action, may follow if we solve the defining relations in terms of zehnbeins and $B$-field. Yet, there exists a class of configurations which do not admit such a Riemannian parametrization even locally at all $[14,37]$ (cf. [51]).

Hereafter, for concreteness, yet without loss of generality, we fix the section as (2.10):

$$
\partial_{M}=\left(\tilde{\partial}^{\mu}, \partial_{\nu}\right) \equiv\left(0, \partial_{\nu}\right), \quad \mathcal{A}_{i M} \equiv\left(0, A_{i \mu}\right) .
$$




\subsection{Type IIA or IIB: Riemannian backgrounds}

The DFT-vielbeins, $V_{M p}$ and $\bar{V}_{M \bar{p}}$, are $20 \times 10$ matrices. If their first half $10 \times 10$ square blocks are non-degenerate, we may parametrize them as $\frac{1}{\sqrt{2}}\left(e^{-1}\right)_{p}{ }^{\mu}$ and $\frac{1}{\sqrt{2}}\left(\bar{e}^{-1}\right)_{\bar{p}}^{\mu}$ respectively with some invertible matrices, $e_{\mu}{ }^{p}$ and $\bar{e}_{\mu}{ }^{\bar{p}}$. Then, the defining relations of the DFT-vielbeins (2.16) determine the remaining $10 \times 10$ blocks with one common free skew-symmetric field, $B_{\mu \nu}=-B_{\nu \mu}[27,36]$,

$$
V_{M p}=\frac{1}{\sqrt{2}}\left(\begin{array}{c}
\left(e^{-1}\right)_{p}{ }^{\mu} \\
(B+e)_{\nu p}
\end{array}\right), \quad \bar{V}_{M \bar{p}}=\frac{1}{\sqrt{2}}\left(\begin{array}{c}
\left(\bar{e}^{-1}\right)_{\bar{p}}{ }^{\mu} \\
(B+\bar{e})_{\nu \bar{p}}
\end{array}\right),
$$

while $e_{\mu}{ }^{p}$ and $\bar{e}_{\nu} \bar{p}$ must meet

$$
e_{\mu}^{p} e_{\nu}^{q} \eta_{p q}=-\bar{e}_{\mu}{ }^{\bar{p}} \bar{e}_{\nu}{ }^{\bar{q}} \bar{\eta}_{\bar{p} \bar{q}}
$$

In (3.2), we set, as usual, $B_{\mu p}=B_{\mu \nu}\left(e^{-1}\right)_{p}{ }^{\nu}, B_{\mu \bar{p}}=B_{\mu \nu}\left(\bar{e}^{-1}\right)_{\bar{p}}{ }^{\nu}$ and $e_{\nu p}=e_{\nu}{ }^{q} \eta_{q p}, \bar{e}_{\nu \bar{p}}=$ $\bar{e}_{\nu} \bar{q}_{\bar{q} \bar{p}}$, etc. Of course, with respect to the choice of the section 3.1, $e_{\mu}^{p}, e_{\nu} \bar{p}$ and $B_{\mu \nu}$ are identified as a pair of zehnbeins corresponding to the common Riemannian metric, $g_{\mu \nu}$, and the NS-NS B-field. It follows that the DFT-metric, or "the generalized metric", is of the well-known form,

$$
\mathcal{H}_{M N}=V_{M p} V_{N}^{p}-\bar{V}_{M \bar{p}} \bar{V}_{N}^{\bar{p}}=P_{M N}-\bar{P}_{M N}=\left(\begin{array}{cc}
g^{-1} & -g^{-1} B \\
B g^{-1} & g-B g^{-1} B
\end{array}\right) .
$$

This is the most general parametrization of the DFT-metric satisfying the defining property (1.8), i.e. 'a symmetric $\mathrm{O}(D, D)$ element', if the upper left $D \times D$ block is invertible.

The existence of the pair of zehnbeins reflects the very fact that the local Lorentz symmetry in DFT is twofold, ${ }^{5}$ i.e. $\operatorname{Spin}(1,9) \times \operatorname{Spin}(9,1)$. It follows that $\left(e^{-1} \bar{e}\right)_{p} \bar{p}$ is a Lorentz rotation,

$$
\left(e^{-1} \bar{e}\right)_{p}^{\bar{p}}\left(e^{-1} \bar{e}\right)_{q} \bar{q}_{\bar{p} \bar{q}}=-\eta_{p q}
$$

and in particular,

$$
\operatorname{det}\left(e^{-1} \bar{e}\right)= \pm 1
$$

Now, assuming the Riemannian parametrization of (3.2), from (A.10), our master Lagrangian (2.2) reduces to

$$
\begin{aligned}
\mathcal{L}_{\mathrm{IIA} / \mathrm{IIB}}= & -\frac{1}{2} \sqrt{-h} h^{i j}\left[\stackrel{\circ}{\Pi}_{i}^{\mu} \stackrel{\circ}{\Pi}_{j}^{\nu} g_{\mu \nu}+\left(\stackrel{\circ}{\Pi}_{i \mu}-A_{i \mu}+\stackrel{\circ}{\Pi}_{i}^{\lambda} B_{\lambda \mu}\right)\left(\stackrel{\circ}{\Pi}_{j \nu}-A_{j \nu}+\stackrel{\circ}{\Pi}_{j}^{\rho} B_{\rho \nu}\right) g^{\mu \nu}\right] \\
& -\epsilon^{i j}\left(\stackrel{\circ}{\Pi}_{i}^{\mu} A_{j \mu}-i \partial_{i} X^{\mu} \widetilde{\Sigma}_{j \mu}-i \partial_{i} \widetilde{X}_{\mu} \Sigma_{j}^{\mu}\right) \\
= & -\sqrt{-h} h^{i j} \stackrel{\circ}{\Pi}_{i}^{\mu} \stackrel{\circ}{\Pi}_{j}^{\nu} g_{\mu \nu}+2 i \epsilon^{i j} \partial_{i} X^{\mu} \widetilde{\Sigma}_{j \mu}+\epsilon^{i j} \Sigma_{i}^{\mu} \widetilde{\Sigma}_{j \mu}+\epsilon^{i j} \stackrel{\circ}{\Pi}_{i}^{\mu} \stackrel{\circ}{\Pi}_{j}^{\nu} B_{\mu \nu}+\epsilon^{i j} \partial_{i} \widetilde{X}_{\mu} \partial_{j} X^{\mu} \\
& -\frac{1}{2} \sqrt{-h} h^{i j}\left(\stackrel{\circ}{\Pi}_{i \mu}+\stackrel{\circ}{\Pi}_{i}^{\lambda} B_{\lambda \mu}+\frac{\epsilon_{i}^{k}}{\sqrt{-h}} \stackrel{\circ}{\Pi}_{k}^{\lambda} g_{\lambda \mu}-A_{i \mu}\right) \\
& \times\left(\stackrel{\circ}{\widetilde{\Pi}}_{j \nu}+\stackrel{\circ}{\Pi}_{j}^{\rho} B_{\rho \nu}+\frac{\epsilon_{j}^{l}}{\sqrt{-h}} \stackrel{\circ}{\Pi}_{l}^{\rho} g_{\rho \nu}-A_{j \nu}\right) g^{\mu \nu}
\end{aligned}
$$

\footnotetext{
${ }^{5}$ The fact that the spin group is twofold can lead to a phenomenological prediction to the Standard Model: the quarks and the leptons may belong to the distinct spin groups [31].
} 
where we put, like (2.45),

$$
\Sigma_{i}^{M}=\left(\widetilde{\Sigma}_{i \mu}, \Sigma_{i}{ }^{\nu}\right),
$$

and we set without the coordinate gauge symmetry potential,

$$
\stackrel{\circ}{\Pi}_{i}^{M}:=\partial_{i} X^{M}-i \Sigma_{i}^{M}=\left(\stackrel{\circ}{\Pi}_{i \mu}, \stackrel{\circ}{\Pi}_{i}^{\nu}\right), \quad \stackrel{\circ}{\Pi}_{i \mu}=\partial_{i} \widetilde{X}_{\mu}-i \widetilde{\Sigma}_{i \mu}, \quad \stackrel{\circ}{\Pi}_{i}^{\mu}=\partial_{i} X^{\mu}-i \Sigma_{i}^{\mu} .
$$

The on-shell value of the coordinate gauge symmetry potential is, from the last line of (3.7) which is a 'perfect square' of the potential,

$$
A_{i \mu} \equiv \stackrel{\circ}{\Pi}_{i \mu}+\stackrel{\circ}{\Pi}_{i}^{\lambda} B_{\lambda \mu}+\frac{\epsilon_{i}{ }^{k}}{\sqrt{-h}} \stackrel{\circ}{\Pi}_{k}^{\lambda} g_{\lambda \mu} .
$$

Therefore, after the auxiliary potential being integrated out, our action reduces to

$$
\begin{aligned}
\mathcal{S}_{\text {IIA } / \mathrm{IIB}}= & \frac{1}{2 \pi \alpha^{\prime}} \int \mathrm{d}^{2} \sigma-\frac{1}{2} \sqrt{-h} h^{i j} \stackrel{\circ}{\Pi}_{i}^{\mu} \stackrel{\circ}{\Pi}_{j}^{\nu} g_{\mu \nu}+\epsilon^{i j}\left(i \partial_{i} X^{\mu}+\frac{1}{2} \Sigma_{i}^{\mu}\right) \widetilde{\Sigma}_{j \mu} \\
& +\frac{1}{2} \epsilon^{i j} \stackrel{\circ}{\Pi}_{i}^{\mu} \stackrel{\circ}{\Pi}_{j}^{\nu} B_{\mu \nu}+\frac{1}{2} \epsilon^{i j} \partial_{i} \widetilde{X}_{\mu} \partial_{j} X^{\mu}
\end{aligned}
$$

where the standard string tension, $\left(2 \pi \alpha^{\prime}\right)^{-1}$, is restored. The last term, as total derivative, is the topological term introduced in [52] and [8].

In order to compare with the original Green-Schwarz action, $i)$ we perform a $\operatorname{Pin}(9,1)$ rotation to let

$$
e_{\mu}^{p} \equiv \bar{e}_{\mu}^{\bar{p}},
$$

ii) truncate the twofold Lorentz symmetry to the diagonal subgroup,

$$
\operatorname{Spin}(1,9) \times \operatorname{Spin}(9,1) \quad \Longrightarrow \quad \operatorname{Spin}(1,9)_{\text {Diagonal }},
$$

and iii) do not distinguish the unbarred and barred spin group indices anymore: in particular, we may identify

$$
\eta_{p q} \equiv-\bar{\eta}_{\bar{p} \bar{q}}, \quad \bar{\gamma}^{\bar{p}} \equiv \gamma^{(11)} \gamma^{p}, \quad \bar{\gamma}^{(11)} \equiv-\gamma^{(11)}
$$

Then depending on the sign value of $\operatorname{det}\left(e^{-1} \bar{e}\right)$ prior to the $\operatorname{Pin}(9,1)$ rotation for $(3.12)$, the conventional classification of type IIA and type IIB can be recovered [30],

$$
\begin{aligned}
& \text { type IIA for } \operatorname{det}\left(e^{-1} \bar{e}\right)=+1, \\
& \text { type IIB for } \operatorname{det}\left(e^{-1} \bar{e}\right)=-1
\end{aligned}
$$

Essentially, when $\operatorname{det}\left(e^{-1} \bar{e}\right)=+1$ we can ensure the identification (3.12) using $\operatorname{Spin}(9,1)$ group without flipping the chirality of $\bar{\theta}^{\prime}$, but when $\operatorname{det}\left(e^{-1} \bar{e}\right)=-1$ we have to use a chirality flipping Pin $(9,1)$ rotation.

In this way, setting $B_{\mu \nu}=0$, up to the world-sheet topological term and constant rescaling of the fermions, $\theta, \bar{\theta} \rightarrow \sqrt[4]{2} \theta, \sqrt[4]{2} \bar{\theta}^{\prime}$, the reduced action (3.11) can be identified as the original undoubled Green-Schwarz superstring action. 
Self-duality over the entire doubled-yet-gauged spacetime. $\quad$ Since $\Pi_{i}^{\mu}=\Pi_{i}^{\mu},(3.10)$ is equivalent to

$$
g^{\mu \nu} \widetilde{\Pi}_{i \nu}-\left(g^{-1} B\right)^{\mu}{ }_{\nu} \Pi_{i}^{\nu}+\frac{\epsilon_{i}{ }^{j}}{\sqrt{-h}} \Pi_{j}^{\mu}=0 .
$$

This gives, contracting with $B_{\lambda \mu}$,

$$
\left(B g^{-1}\right)_{\lambda}{ }^{\nu} \widetilde{\Pi}_{i \nu}-\left(B g^{-1} B\right)_{\lambda \nu} \Pi_{i}^{\nu}+\frac{\epsilon_{i}^{j}}{\sqrt{-h}} B_{\lambda \mu} \Pi_{j}^{\mu}=0,
$$

and further separately, contracting with $g_{\lambda \mu} \frac{\epsilon_{k}{ }^{i}}{\sqrt{-h}}$,

$$
\frac{\epsilon_{i}{ }^{j}}{\sqrt{-h}}\left(\widetilde{\Pi}_{j \lambda}-B_{\lambda \nu} \Pi_{j}^{\nu}\right)+g_{\lambda \nu} \Pi_{i}^{\nu}=0 .
$$

Adding (3.17) and (3.18), we obtain

$$
\left(B g^{-1}\right)_{\lambda} \widetilde{\Pi}_{i \nu}+\left(g-B g^{-1} B\right)_{\lambda \nu} \Pi_{i}^{\nu}+\frac{\epsilon_{i}{ }^{j}}{\sqrt{-h}} \widetilde{\Pi}_{j \lambda}=0 .
$$

Then, as in the case with the bosonic string action of [14], (3.16) and (3.19) imply that the full set of the self-duality relations hold over the entire doubled-yet-gauged spacetime coordinate directions - although the coordinate gauge symmetry is a constrained field when the NS-NS background is Riemannian,

$$
\widehat{\Pi}_{i M}=\Pi_{i M}+\frac{\epsilon_{i}^{j}}{\sqrt{-h}} \mathcal{H}_{M}{ }^{N} \Pi_{j N}=\left(\widehat{\Pi}_{i}^{\mu}, \widetilde{\Pi}_{i \nu}\right)=0 .
$$

In the generic cases, i.e. not necessarily Riemannian, the equation of motion of the coordinate gauge symmetry potential gives a priori only the half of the self-duality relations, from (2.33),

$$
\epsilon^{i j} \delta \mathcal{A}_{i M} \widehat{\Pi}_{j}^{M}=0 \quad \Longrightarrow \quad \widehat{\Pi}_{i}^{\mu}=0 .
$$

Then the above result (3.20) tells us that when the NS-NS background admits Riemannian interpretation, the other half of the self-duality relations is automatically satisfied, $\widetilde{\widehat{\Pi}}_{i \nu}=0$. It is useful to note that, contracting with the DFT-vielbeins, the self-duality (3.20) decomposes into

$$
h_{+}^{i j} \Pi_{j p}=0, \quad h_{-}^{i j} \Pi_{j \bar{p}}=0 .
$$

The self-dual part of $\Pi_{i}^{M}$ satisfies, from (A.3), (A.4),

$$
\widehat{\Pi}_{i M}=\frac{\epsilon_{i}^{j}}{\sqrt{-h}} \mathcal{H}_{M}{ }^{N} \widehat{\Pi}_{j N}, \quad \epsilon^{i j} \widehat{\Pi}_{j M}=\epsilon^{i j} \Pi_{j M}+\sqrt{-h} h^{i j} \mathcal{H}_{M}{ }^{N} \Pi_{j N} .
$$

\subsection{Type IIC: non-Riemannian and non-relativistic backgrounds}

While the non-Riemannian NS-NS background was first noted in [14] and subsequently shown in [37] to lead to the Gomis-Ooguri non-relativistic bosonic string [38], until now there is no systematic classification of it. Decomposing the DFT-vielbeins in terms of $10 \times 10$ square matrices, such as $V_{M p}=\left(V_{p}^{\mu}, \widetilde{V}_{\nu p}\right)$ and $\bar{V}_{M \bar{p}}=\left(\bar{V}_{\bar{p}}^{\mu}, \widetilde{\bar{V}}_{\nu \bar{p}}\right)$, the defining relations of them (2.16), especially the last one, imply

$$
V_{p}^{\mu} V_{q}^{\nu} \eta^{p q}=-\bar{V}_{\bar{p}}^{\mu} \bar{V}_{\bar{q}}^{\nu} \bar{\eta}^{\bar{p} \bar{q}}
$$

This shows that $V_{p}^{\mu}$ is invertible if and only if $\bar{V}_{\bar{p}}^{\mu}$ is so. 
In this subsection, we focus on the non-Riemannian background for the Gomis-Ooguri non-relativistic string. For this, we need to decompose $\mathrm{O}(10,10)$ into $\mathrm{O}(2,2) \times \mathrm{O}(8,8)$, such that the doubled coordinates decompose as $x^{M}=\left(x^{\hat{M}}, x^{M^{\prime}}\right)$, where including the time directions we set $x^{\hat{M}}=\left(\tilde{x}_{\hat{\mu}}, x^{\hat{\nu}}\right)=\left(\tilde{t}, \tilde{x}_{1}, t, x^{1}\right)$, while $x^{M^{\prime}}$ denotes the remaining 16 spatial part of the doubled-yet-gauged coordinates. With respect to the choice of the section 3.1, the DFT-metric for the non-Riemannian NS-NS background reads [14]

$$
\mathcal{H}_{M N}=\left(\begin{array}{cccc}
0 & 0 & \hat{\epsilon}^{\hat{\lambda}} \hat{\nu} & 0 \\
0 & \delta^{\lambda^{\prime} \rho^{\prime}} & 0 & 0 \\
-\hat{\epsilon}_{\hat{\mu}} \hat{\rho} & 0 & \hat{\eta}_{\hat{\mu} \hat{\nu}} & 0 \\
0 & 0 & 0 & \delta_{\mu^{\prime} \nu^{\prime}}
\end{array}\right)
$$

where we set a two-dimensional flat Minkowskian metric, $\hat{\eta}_{\hat{\mu} \hat{\nu}}=\operatorname{diag}(-+)$, and a skewsymmetric Levi-Civita symbol, $\hat{\epsilon}_{\hat{\mu} \hat{\nu}}$, with $\hat{\epsilon}_{\hat{\nu}} \hat{\nu}_{\hat{\nu}} \hat{\eta}^{\hat{\lambda} \hat{\mu}} \hat{\epsilon}_{\hat{\mu} \hat{\nu}}, \hat{\epsilon}_{\hat{\mu}} \hat{\rho}=\hat{\epsilon}_{\hat{\mu} \hat{\nu}} \hat{\eta}^{\hat{\nu} \hat{\rho}}$, etc. The upper left $2 \times 2$ block is vanishing completely and hence clearly non-Riemannian in nature, in contrast to the Riemannian generalized metric (3.4).

The corresponding DFT-vielbeins are essentially,

$$
\hat{V}_{\hat{M} \hat{p}}=\frac{1}{\sqrt{2}}\left(\begin{array}{rr}
1 & -1 \\
-1 & 1 \\
-1 & 0 \\
0 & 1
\end{array}\right), \quad \hat{\bar{V}}_{\hat{M} \hat{p}}=\frac{1}{\sqrt{2}}\left(\begin{array}{rr}
1 & 1 \\
1 & 1 \\
1 & 0 \\
0 & -1
\end{array}\right) .
$$

As $4 \times 2$ matrices, these represent the genuinely non-Riemannian 'hatted' part of the full DFT-vielbeins. The remaining 16 doubled-yet-gauged 'primed' coordinates is flat Riemannian: assigned the flat Euclidean kronecker-delta symbol, $\delta_{\mu^{\prime} \nu^{\prime}}$, as the spacetime metric with constant $B$-field.

The master Lagrangian (2.2) reduces, upon the non-Riemannian NS-NS background, to

$$
\begin{aligned}
& \mathcal{L}_{\mathrm{IIC}}=-\sqrt{-h} h^{i j} \stackrel{\circ}{\Pi}_{i}^{\mu} \stackrel{\circ}{\Pi}_{j}^{\nu} \stackrel{\circ}{g \nu}_{\mu \nu}+\epsilon^{i j}\left(2 i \partial_{i} X^{\mu}+\Sigma_{i}^{\mu}\right) \widetilde{\Sigma}_{j \mu}+\epsilon^{i j} \partial_{i} \widetilde{X}_{\mu} \partial_{j} X^{\mu}+\epsilon^{i j} \stackrel{\circ}{\Pi}_{i}^{\mu^{\prime}} \stackrel{\circ}{\Pi}_{j}^{\nu^{\prime}} B_{\mu^{\prime} \nu^{\prime}} \\
& +\sqrt{-h}\left(\mathcal{A}_{i \hat{\mu}}-\stackrel{\widetilde{\Pi}}{i \hat{\mu}}\right)\left(h^{i j} \hat{\epsilon}_{\hat{\mu}}^{\hat{\nu}} \stackrel{\circ}{\Pi}_{j}^{\hat{\nu}}+\frac{\epsilon^{i j}}{\sqrt{-h}} \stackrel{\circ}{\Pi}_{j}^{\hat{\mu}}\right) \\
& -\frac{1}{2} \sqrt{-h}\left\|\stackrel{\circ}{\Pi}_{i \mu^{\prime}}+\stackrel{\circ}{\Pi}_{i}^{\lambda^{\prime}} B_{\lambda^{\prime} \mu^{\prime}}+\frac{\epsilon_{i}^{k}}{\sqrt{-h}} \stackrel{\circ}{\Pi}_{k}^{\lambda^{\prime}}{\stackrel{\circ}{\lambda^{\prime} \mu^{\prime}}}-A_{i \mu^{\prime}}\right\|^{2},
\end{aligned}
$$

where we set a ten-dimensional target-spacetime constant metric,

$$
\stackrel{\circ}{g}_{\mu \nu}:=\left(\frac{1}{2} \hat{\eta}_{\hat{\mu} \hat{\nu}}, \delta_{\mu^{\prime} \nu^{\prime}}\right) \text {. }
$$

The resulting superstring action is then,

$$
\begin{aligned}
\mathcal{S}_{\text {IIC }}= & \frac{1}{2 \pi \alpha^{\prime}} \int \mathrm{d}^{2} \sigma-\frac{1}{2} \sqrt{-h} h^{i j} \stackrel{\circ}{\Pi}_{i}^{\mu} \stackrel{\circ}{\Pi}_{j}^{\nu} \stackrel{\circ}{g}_{\mu \nu}+\epsilon^{i j}\left(i \partial_{i} X^{\mu}+\frac{1}{2} \Sigma_{i}^{\mu}\right) \widetilde{\Sigma}_{j \mu} \\
& +\frac{1}{2} \epsilon^{i j} \partial_{i} \widetilde{X}_{\mu} \partial_{j} X^{\mu}+\frac{1}{2} \epsilon^{i j} \stackrel{\circ}{\Pi}_{i}^{\mu^{\prime}} \stackrel{\circ}{\Pi}_{j}^{\nu^{\prime}} B_{\mu^{\prime} \nu^{\prime}},
\end{aligned}
$$

and is subject to the chirality condition for the hatted untilde directions, $x^{\hat{\mu}}=\left(t, x^{1}\right)$ :

$$
\stackrel{\circ}{\Pi}_{i}^{\hat{\mu}}+\frac{\epsilon_{i}^{j}}{\sqrt{-h}} \hat{\epsilon}_{\hat{\nu}}^{\hat{\mu}} \stackrel{\circ}{\Pi}_{j}^{\hat{\nu}}=0 .
$$

This is the action for the Green-Schwarz superstring on the non-Riemannian background which supersymmetrizes the Gomis-Ooguri non-relativistic string. 


\section{Discussion}

In this work, we have constructed a world-sheet action for Green-Schwarz superstring which propagates on doubled-yet-gauged spacetime. For an arbitrarily curved NS-NS background, the action possesses manifest $\mathrm{O}(10,10) \mathrm{T}$-duality, $\operatorname{Spin}(1,9) \times \operatorname{Spin}(9,1)$ global Lorentz symmetry, coordinate gauge symmetry, target-spacetime doubled-yet-gauged diffeomorphisms, world-sheet diffeomorphisms and Weyl symmetry. Restricted to flat backgrounds of constant DFT-vielbeins, the action is further invariant under maximal spacetime global supersymmetry and also under local fermionic kappa-symmetry. After the auxiliary coordinate gauge symmetry potential being integrated out, the action can consistently reduce to the undoubled original Green-Schwarz action upon a Riemannian background. Thanks to the twofold spin groups, the action is unique: the two fermions, $\theta^{\alpha}$ and $\theta^{\prime \bar{\alpha}}$, are Majora-Weyl spinors for $\operatorname{Spin}(1,9)$ and $\operatorname{Spin}(9,1)$ respectively. It is then specific choices of the NS-NS backgrounds that distinguish Riemannian IIA, IIB and non-Riemannian IIC. Upon the Riemmanian IIA/IIB backgrounds, the Euler-Lagrangian equation of the coordinate gauge symmetry potential implies the self-duality over the entire doubled-yet-gauged spacetime.

It will be of interest to couple our action to the $\operatorname{Spin}(1,9) \times \operatorname{Spin}(9,1)$ bi-spinorial $\mathrm{R}-\mathrm{R}$ sector [36]. Investigating the supersymmetry, the Killing spinor equations of the maximally supersymmetric DFT [30] should appear naturally. The computations of the one-loop beta function and the partition function are worth while to perform: we expect to derive the equations of motion of the maximally supersymmetric DFT [30]. Related to this, we refer readers to earlier works [53-55] on bosonic doubled sigma models, along with [37] for the matching of the fluctuation spectrum between DFT and the bosonic worldsheet action (1.14) around the non-Riemannian background for the Gomis-Ooguri string. Promoting the global $\operatorname{Spin}(1,9) \times \operatorname{Spin}(9,1)$ Lorentz symmetry to the local symmetry seems desirable. We leave quantization as for future work.

\section{Acknowledgments}

We wish to thank Kanghoon Lee, Charles M. Melby-Thompson and René Meyer for discussions. We also thank an anonymous referee for suggesting us to look for the Wess-Zumino term (2.27). This work was supported by the National Research Foundation of Korea with Grant Nos. 2015K1A3A1A21000302 and 2016R1D1A1B0101519.

\section{A Useful identities}

In addition to (2.31), the Fierz identity (2.25) implies for arbitrary $\delta \theta$ and $\delta \theta^{\prime}$,

$$
\begin{aligned}
& \epsilon^{i j} \partial_{i}\left(\bar{\theta} \gamma^{p} \delta \theta\right) \bar{\theta} \gamma_{p} \partial_{j} \theta=\frac{1}{3} \partial_{i}\left(\epsilon^{i j} \bar{\theta} \gamma^{p} \delta \theta \bar{\theta} \gamma_{p} \partial_{j} \theta\right)+\frac{2}{3} \epsilon^{i j} \bar{\theta} \gamma^{p}\left(\partial_{i} \delta \theta\right) \bar{\theta} \gamma_{p} \partial_{j} \theta, \\
& \epsilon^{i j} \partial_{i}\left(\bar{\theta}^{\prime} \bar{\gamma}^{\bar{p}} \delta \theta^{\prime}\right) \bar{\theta}^{\prime} \bar{\gamma}_{\bar{p}} \partial_{j} \theta^{\prime}=\frac{1}{3} \partial_{i}\left(\epsilon^{i j} \bar{\theta}^{\prime} \bar{\gamma}^{\bar{p}} \delta \theta^{\prime} \bar{\theta}^{\prime} \bar{\gamma}_{\bar{p}} \partial_{j} \theta^{\prime}\right)+\frac{2}{3} \epsilon^{i j} \bar{\theta}^{\prime} \bar{\gamma}^{\bar{p}}\left(\partial_{i} \delta \theta^{\prime}\right) \bar{\theta}^{\prime} \bar{\gamma}_{\bar{p}} \partial_{j} \theta^{\prime},
\end{aligned}
$$

and

$$
\begin{aligned}
\epsilon^{i j} \bar{\theta} \gamma^{p}\left(\partial_{i} \delta \theta\right) \bar{\theta} \gamma_{p} \partial_{j} \theta & =\partial_{i}\left(\epsilon^{i j} \bar{\theta} \gamma^{p} \delta \theta \bar{\theta} \gamma_{p} \partial_{j} \theta\right)+3 \epsilon^{i j} \delta \bar{\theta} \gamma^{p} \partial_{i} \theta \bar{\theta} \gamma_{p} \partial_{j} \theta \\
\epsilon^{i j} \bar{\theta}^{\prime} \bar{\gamma}^{\bar{p}}\left(\partial_{i} \delta \theta^{\prime}\right) \bar{\theta}^{\prime} \bar{\gamma}_{\bar{p}} \partial_{j} \theta^{\prime} & =\partial_{i}\left(\epsilon^{i j} \bar{\theta}^{\prime} \bar{\gamma}^{\bar{p}} \delta \theta^{\prime} \bar{\theta}^{\prime} \bar{\gamma}_{\bar{p}} \partial_{j} \theta^{\prime}\right)+3 \epsilon^{i j} \delta \bar{\theta}^{\prime} \bar{\gamma}^{\bar{p}} \partial_{i} \theta^{\prime} \bar{\theta}^{\prime} \bar{\gamma}_{\bar{p}} \partial_{j} \theta^{\prime}
\end{aligned}
$$


It is worth while to note

$$
\begin{array}{rlrl}
\epsilon^{i j} \epsilon^{k l} & =h\left(h^{i k} h^{j l}-h^{j k} h^{i l}\right), & \epsilon_{i j}^{-1} \epsilon_{k l}^{-1} & =h^{-1}\left(h_{i k} h_{j l}-h_{j k} h_{i l}\right), \\
\epsilon^{i}{ }_{j} \epsilon^{j}{ }_{k} & =\epsilon^{i l} \epsilon^{j m} h_{l j} h_{m k}=-h \delta^{i}{ }_{k}, & \epsilon_{i j}^{-1} & =-h^{-1} h_{i k} h_{j l} \epsilon^{k l}=-h^{-1} \epsilon_{i j}, \\
h_{ \pm j}^{i}{\frac{\epsilon^{j k}}{\sqrt{-h}}}^{k l} & =h_{ \pm j}^{i}\left(h_{+}^{j k}-h_{-}^{j k}\right)= \pm h_{ \pm}^{i k}, & \sqrt{-h} h_{ \pm}^{i j} \epsilon_{j k}^{-1} & = \pm h_{ \pm k}^{i}, \\
\frac{\epsilon^{i j}}{\sqrt{-h}} h_{ \pm j}{ }^{k} & = \pm h_{ \pm}^{i k}, \\
h_{ \pm}^{i j} h_{ \pm}^{k l} & =h_{ \pm}^{i l} h_{ \pm}^{k j}, & \sqrt{-h} \epsilon_{i j}^{-1} h_{ \pm}^{j k} & = \pm h_{ \pm i}{ }^{k}, \\
h_{ \pm i}^{i} & =1, & h_{ \pm}^{i k} h_{ \pm}^{j l}\left(\epsilon_{k}{ }^{m} \delta_{l}{ }^{n}-\epsilon_{l}{ }^{n} \delta_{k}{ }^{m}\right) & =0, \\
\operatorname{det}\left(h_{ \pm}^{i j}\right) & =0,
\end{array}
$$

and

$$
\gamma_{p q} \Pi_{i}^{p} \Pi_{j}^{q}=-\frac{1}{2} \epsilon_{i j}^{-1} \epsilon^{k l} \gamma_{p q} \Pi_{k}^{p} \Pi_{l}^{q}, \bar{\gamma}_{\bar{p} \bar{q}} \Pi_{i}^{\bar{p}} \Pi_{j}^{\bar{q}}=-\frac{1}{2} \epsilon_{i j}^{-1} \epsilon^{k l} \bar{\gamma}_{\bar{p} \bar{q}} \Pi_{k}^{\bar{p}} \Pi_{l}^{\bar{q}} .
$$

Substituting the ansatz (2.35) into (2.33), the variation of the Lagrangian reduces to

$$
\begin{aligned}
\delta \mathcal{L}_{\text {superstring }} & \\
= & -\frac{1}{2}\left[\delta\left(\sqrt{-h} h^{i j}\right)+4 i \sqrt{-h}\left(\partial_{k} \bar{\theta} \zeta^{(i} h_{+}^{j j k}+\partial_{k} \bar{\theta}^{\prime} \zeta^{\prime(i} h_{-}^{j) k}\right)\right] \Pi_{i}^{M} \Pi_{j}^{N} \mathcal{H}_{M N} \\
& -2 i \sqrt{-h}\left(\partial_{k} \bar{\theta} \zeta^{(i} h_{+}^{j) k}-\partial_{k} \bar{\theta}^{\prime} \zeta^{\prime(i} h_{-}^{j) k}\right) \Pi_{i M} \Pi_{j}^{M}+\left(\sqrt{-h} h^{i j} \mathcal{H}^{M}{ }_{N} \Pi_{j}^{N}+\epsilon^{i j} \Pi_{j}^{M}\right) \delta \mathcal{A}_{i M} \\
& -2 i\left(h_{-j}^{i} \partial_{i} \bar{\theta} \gamma_{M N} \zeta^{j}+h_{+j}^{i} \partial_{i} \bar{\theta}^{\prime} \bar{\gamma}_{M N} \zeta^{\prime j}\right)\left(\epsilon^{k l} \Pi_{k}^{M} \Pi_{l}^{N}\right)-\partial_{i}\left[\epsilon^{i j}\left(\Pi_{j}{ }^{M}+\mathcal{A}_{j}^{M}\right) \delta X_{M}\right] \\
= & -\frac{1}{2}\left[\delta\left(\sqrt{-h} h^{i j}\right)+8 i \sqrt{-h}\left(\partial_{k} \bar{\theta} \zeta^{(i} h_{+}^{j) k}+\partial_{k} \bar{\theta}^{\prime} \zeta^{\prime(i} h_{-}^{j) k}\right)\right] \Pi_{i}^{M} \Pi_{j}^{N} \mathcal{H}_{M N} \\
& +\left(\sqrt{-h} h^{i j} \mathcal{H}^{M}{ }_{N} \Pi_{j}^{N}+\epsilon^{i j} \Pi_{j}^{M}\right)\left[\delta \mathcal{A}_{i M}+2 i\left(h_{+i}{ }^{k} \partial_{k} \bar{\theta} \zeta^{l}+h_{-i}{ }^{k} \partial_{k} \bar{\theta}^{\prime} \zeta^{\prime l}\right) \Pi_{l M}\right] \\
& -2 i\left(h_{-j}^{i} \partial_{i} \bar{\theta} \gamma_{M N} \zeta^{j}+h_{+j}^{i} \partial_{i} \bar{\theta}^{\prime} \bar{\gamma}_{M N} \zeta^{\prime j}\right)\left(\epsilon^{k l} \Pi_{k}^{M} \Pi_{l}^{N}\right)-\partial_{i}\left[\epsilon^{i j}\left(\Pi_{j}{ }^{M}+\mathcal{A}_{j}^{M}\right) \delta X_{M}\right]
\end{aligned}
$$

This further becomes, with (2.37) and (2.38),

$$
\begin{aligned}
& \delta \mathcal{L}_{\text {superstring }}+\partial_{i}\left[\epsilon^{i j}\left(\Pi_{j}^{M}+\mathcal{A}_{j}^{M}\right) \delta X_{M}\right] \\
& =\left[\epsilon^{i j} \delta \mathcal{A}_{i M}+2 i \sqrt{-h}\left(-h_{+}^{j k} h_{+}^{l m} \partial_{k} \bar{\theta} \kappa_{m}+h_{-}^{j k} h_{-}^{l m} \partial_{k} \bar{\theta}^{\prime} \kappa_{m}^{\prime}\right) \Pi_{l M}\right] \widehat{\Pi}_{j}^{M} \\
& =\left[\epsilon^{i j} \delta \mathcal{A}_{i M}+i \sqrt{-h}\left(-h_{+}^{j k} h_{+}^{l m} \partial_{k} \bar{\theta} \kappa_{m}+h_{-}^{j k} h_{-}^{l m} \partial_{k} \bar{\theta}^{\prime} \kappa_{m}^{\prime}\right) \widehat{\Pi}_{l M}\right] \widehat{\Pi}_{j}^{M} \\
& =\epsilon^{i j}\left[\delta \mathcal{A}_{i M}+i\left(h_{+i}{ }^{k} h_{+}^{l m} \partial_{k} \bar{\theta} \kappa_{m}+h_{-i}{ }^{k} h_{-}^{l m} \partial_{k} \bar{\theta}^{\prime} \kappa_{m}^{\prime}\right) \widehat{\Pi}_{l M}\right] \widehat{\Pi}_{j}^{M},
\end{aligned}
$$

where for the second equality we have used identities,

$$
h_{+}^{j k} h_{+}^{l m} \widehat{\Pi}_{j M} \Pi_{l}^{M}=\frac{1}{2} h_{+}^{j k} h_{+}^{l m} \widehat{\Pi}_{j M} \widehat{\Pi}_{l}^{M}, \quad h_{-}^{j k} h_{-}^{l m} \widehat{\Pi}_{j M} \Pi_{l}^{M}=\frac{1}{2} h_{-}^{j k} h_{-}^{l m} \widehat{\Pi}_{j M} \widehat{\Pi}_{l}^{M} .
$$

One useful relation to establish the second equality in (3.7) is

$$
\begin{aligned}
- & \frac{1}{2} \sqrt{-h} h^{i j}\left(\tilde{Y}_{i \mu}-A_{i \mu}\right)\left(\tilde{Y}_{j \nu}-A_{j \nu}\right) g^{\mu \nu}-\epsilon^{i j} Z_{i}^{\mu} A_{j \mu} \\
= & -\frac{1}{2} \sqrt{-h} h^{i j}\left(\frac{\epsilon_{i}{ }^{k}}{\sqrt{-h}} Z_{k}{ }^{\lambda} g_{\lambda \mu}+\tilde{Y}_{i \mu}-A_{i \mu}\right)\left(\frac{\epsilon_{j}^{l}}{\sqrt{-h}} Z_{l}^{\rho} g_{\rho \nu}+\tilde{Y}_{j \nu}-A_{j \nu}\right) g^{\mu \nu} \\
& -\frac{1}{2} \sqrt{-h} h^{i j} Z_{i}^{\mu} Z_{j}^{\nu} g_{\mu \nu}-\epsilon^{i j} Z_{i}^{\mu} \tilde{Y}_{j \mu} .
\end{aligned}
$$

This identity is true for arbitrary $\tilde{Y}_{i \mu}$ and $Z_{j}{ }^{\nu}$. 
Open Access. This article is distributed under the terms of the Creative Commons Attribution License (CC-BY 4.0), which permits any use, distribution and reproduction in any medium, provided the original author(s) and source are credited.

\section{References}

[1] T.H. Buscher, A Symmetry of the String Background Field Equations, Phys. Lett. B 194 (1987) 59 [INSPIRE].

[2] T.H. Buscher, Path Integral Derivation of Quantum Duality in Nonlinear $\sigma$-models, Phys. Lett. B 201 (1988) 466 [INSPIRE].

[3] M.J. Duff, Duality Rotations in String Theory, Nucl. Phys. B 335 (1990) 610 [InSPIRE].

[4] A.A. Tseytlin, Duality Symmetric Formulation of String World Sheet Dynamics, Phys. Lett. B 242 (1990) 163 [INSPIRE].

[5] A.A. Tseytlin, Duality symmetric closed string theory and interacting chiral scalars, Nucl. Phys. B 350 (1991) 395 [InSPIRE].

[6] C.M. Hull, A Geometry for non-geometric string backgrounds, JHEP 10 (2005) 065 [hep-th/0406102] [INSPIRE].

[7] C.M. Hull, Global aspects of T-duality, gauged $\sigma$-models and T-folds, JHEP 10 (2007) 057 [hep-th/0604178] [INSPIRE].

[8] C.M. Hull, Doubled Geometry and T-Folds, JHEP 07 (2007) 080 [hep-th/0605149] [INSPIRE].

[9] W. Siegel, Two vierbein formalism for string inspired axionic gravity, Phys. Rev. D 47 (1993) 5453 [hep-th/9302036] [INSPIRE].

[10] W. Siegel, Superspace duality in low-energy superstrings, Phys. Rev. D 48 (1993) 2826 [hep-th/9305073] [INSPIRE].

[11] C. Hull and B. Zwiebach, Double Field Theory, JHEP 09 (2009) 099 [arXiv:0904.4664] [INSPIRE].

[12] S.M. Ko, J.-H. Park and M. Suh, The rotation curve of a point particle in stringy gravity, arXiv: 1606.09307 [INSPIRE].

[13] J.-H. Park, Comments on double field theory and diffeomorphisms, JHEP 06 (2013) 098 [arXiv: 1304.5946] [INSPIRE].

[14] K. Lee and J.-H. Park, Covariant action for a string in "doubled yet gauged" spacetime, Nucl. Phys. B 880 (2014) 134 [arXiv:1307.8377] [INSPIRE].

[15] D. Geissbuhler, Double Field Theory and $N=4$ Gauged Supergravity, JHEP 11 (2011) 116 [arXiv: 1109.4280] [INSPIRE].

[16] M. Graña and D. Marques, Gauged Double Field Theory, JHEP 04 (2012) 020 [arXiv: 1201.2924] [INSPIRE].

[17] W. Cho, J.J. Fernández-Melgarejo, I. Jeon and J.-H. Park, Supersymmetric gauged double field theory: systematic derivation by virtue of twist, JHEP 08 (2015) 084 [arXiv: 1505.01301] [INSPIRE].

[18] C. Hull and B. Zwiebach, The Gauge algebra of double field theory and Courant brackets, JHEP 09 (2009) 090 [arXiv: 0908.1792] [INSPIRE]. 
[19] M. Gualtieri, Generalized complex geometry, math/0401221 [INSPIRE].

[20] M. Graña, R. Minasian, M. Petrini and D. Waldram, T-duality, Generalized Geometry and Non-Geometric Backgrounds, JHEP 04 (2009) 075 [arXiv:0807.4527] [INSPIRE].

[21] O. Hohm and B. Zwiebach, Large Gauge Transformations in Double Field Theory, JHEP 02 (2013) 075 [arXiv: 1207.4198] [INSPIRE].

[22] D.S. Berman, M. Cederwall and M.J. Perry, Global aspects of double geometry, JHEP 09 (2014) 066 [arXiv:1401.1311] [INSPIRE].

[23] C.M. Hull, Finite Gauge Transformations and Geometry in Double Field Theory, JHEP 04 (2015) 109 [arXiv: 1406.7794] [INSPIRE].

[24] N. Chaemjumrus and C.M. Hull, Finite Gauge Transformations and Geometry in Extended Field Theory, Phys. Rev. D 93 (2016) 086007 [arXiv:1512.03837] [INSPIRE].

[25] U. Naseer, A note on large gauge transformations in double field theory, JHEP 06 (2015) 002 [arXiv:1504.05913] [INSPIRE].

[26] S.-J. Rey and Y. Sakatani, Finite Transformations in Doubled and Exceptional Space, arXiv:1510.06735 [INSPIRE].

[27] I. Jeon, K. Lee and J.-H. Park, Stringy differential geometry, beyond Riemann, Phys. Rev. D 84 (2011) 044022 [arXiv:1105.6294] [INSPIRE].

[28] I. Jeon, K. Lee and J.-H. Park, Differential geometry with a projection: Application to double field theory, JHEP 04 (2011) 014 [arXiv: 1011.1324] [INSPIRE].

[29] O. Hohm and B. Zwiebach, On the Riemann Tensor in Double Field Theory, JHEP 05 (2012) 126 [arXiv:1112.5296] [INSPIRE].

[30] I. Jeon, K. Lee, J.-H. Park and Y. Suh, Stringy Unification of Type IIA and IIB Supergravities under $N=2 D=10$ Supersymmetric Double Field Theory, Phys. Lett. B 723 (2013) 245 [arXiv:1210.5078] [INSPIRE].

[31] K.-S. Choi and J.-H. Park, Standard Model as a Double Field Theory, Phys. Rev. Lett. 115 (2015) 171603 [arXiv:1506.05277] [INSPIRE].

[32] I. Jeon, K. Lee and J.-H. Park, Double field formulation of Yang-Mills theory, Phys. Lett. B 701 (2011) 260 [arXiv:1102.0419] [INSPIRE].

[33] I. Jeon, K. Lee and J.-H. Park, Incorporation of fermions into double field theory, JHEP 11 (2011) 025 [arXiv:1109.2035] [INSPIRE].

[34] I. Jeon, K. Lee and J.-H. Park, Supersymmetric Double Field Theory: Stringy Reformulation of Supergravity, Phys. Rev. D 85 (2012) 081501 [Erratum ibid. D 86 (2012) 089903] [arXiv: 1112.0069] [INSPIRE].

[35] O. Hohm, S.K. Kwak and B. Zwiebach, Unification of Type II Strings and T-duality, Phys. Rev. Lett. 107 (2011) 171603 [arXiv:1106.5452] [INSPIRE].

[36] I. Jeon, K. Lee and J.-H. Park, Ramond-Ramond Cohomology and $O(D, D)$ T-duality, JHEP 09 (2012) 079 [arXiv: 1206.3478] [INSPIRE].

[37] S.M. Ko, C. Melby-Thompson, R. Meyer and J.-H. Park, Dynamics of Perturbations in Double Field Theory \& Non-Relativistic String Theory, JHEP 12 (2015) 144 [arXiv: 1508.01121] [INSPIRE]. 
[38] J. Gomis and H. Ooguri, Nonrelativistic closed string theory, J. Math. Phys. 42 (2001) 3127 [hep-th/0009181] [INSPIRE].

[39] M.B. Green and J.H. Schwarz, Covariant Description of Superstrings, Phys. Lett. B 136 (1984) 367 [inSPIRE].

[40] S. Driezen, A. Sevrin and D.C. Thompson, Aspects of the Doubled Worldsheet, arXiv: 1609.03315 [INSPIRE].

[41] M. Hatsuda, K. Kamimura and W. Siegel, Type II chiral affine Lie algebras and string actions in doubled space, JHEP 09 (2015) 113 [arXiv:1507.03061] [INSPIRE].

[42] M. Cederwall, Double supergeometry, JHEP 06 (2016) 155 [arXiv:1603.04684] [INSPIRE].

[43] C.D.A. Blair, E. Malek and A.J. Routh, $A n O(D, D)$ invariant Hamiltonian action for the superstring, Class. Quant. Grav. 31 (2014) 205011 [arXiv: 1308.4829] [INSPIRE].

[44] L. Freidel, R.G. Leigh and D. Minic, Born Reciprocity in String Theory and the Nature of Spacetime, Phys. Lett. B 730 (2014) 302 [arXiv:1307.7080] [INSPIRE].

[45] L. Freidel, R.G. Leigh and D. Minic, Metastring Theory and Modular Space-time, JHEP 06 (2015) 006 [arXiv:1502.08005] [INSPIRE].

[46] I. Bandos, Superstring in doubled superspace, Phys. Lett. B 751 (2015) 408 [arXiv: 1507.07779] [INSPIRE].

[47] I. Bandos, Type II superstring in doubled superspace, Fortsch. Phys. 64 (2016) 361 [INSPIRE].

[48] J. Wess and B. Zumino, Consequences of anomalous Ward identities, Phys. Lett. B 37 (1971) 95 [inSPIRE].

[49] M. Henneaux and L. Mezincescu, A $\sigma$-model Interpretation of Green-Schwarz Covariant Superstring Action, Phys. Lett. B 152 (1985) 340 [INSPIRE].

[50] D. Andriot and A. Betz, $\beta$-supergravity: a ten-dimensional theory with non-geometric fluxes and its geometric framework, JHEP 12 (2013) 083 [arXiv:1306.4381] [INSPIRE].

[51] M. Garcia-Fernandez, Torsion-free generalized connections and Heterotic Supergravity, Commun. Math. Phys. 332 (2014) 89 [arXiv:1304.4294] [INSPIRE].

[52] A. Giveon and M. Roček, Generalized duality in curved string backgrounds, Nucl. Phys. B 380 (1992) 128 [hep-th/9112070] [INSPIRE].

[53] D.S. Berman, N.B. Copland and D.C. Thompson, Background Field Equations for the Duality Symmetric String, Nucl. Phys. B 791 (2008) 175 [arXiv:0708.2267] [INSPIRE].

[54] D.S. Berman and D.C. Thompson, Duality Symmetric Strings, Dilatons and O(d,d) Effective Actions, Phys. Lett. B 662 (2008) 279 [arXiv:0712.1121] [INSPIRE].

[55] H.S. Tan, Closed String Partition Functions in Toroidal Compactifications of Doubled Geometries, JHEP 05 (2014) 133 [arXiv:1403.4683] [INSPIRE]. 